\title{
Thermodynamics of Two-Dimensional Electron Gas in a Magnetic Field
}

\author{
V. I. Nizhankovskii \\ International Laboratory of High Magnetic Fields and Low Temperatures, Gajowicka 95, 53-421 Wroclaw, Poland \\ Correspondence should be addressed to V. I. Nizhankovskii, nizhan@alpha.ml.pan.wroc.pl \\ Received 11 September 2010; Revised 27 December 2010; Accepted 10 January 2011 \\ Academic Editor: Israel Felner \\ Copyright () 2011 V. I. Nizhankovskii. This is an open access article distributed under the Creative Commons Attribution License, \\ which permits unrestricted use, distribution, and reproduction in any medium, provided the original work is properly cited.
}

Change of the chemical potential of electrons in a $\mathrm{GaAs}_{\mathrm{Al}} \mathrm{Al}_{x} \mathrm{Ga}_{1-x}$ As heterojunction was measured in magnetic fields up to $6.5 \mathrm{~T}$ at several temperatures from 2.17 to $12.3 \mathrm{~K}$. A thermodynamic equation of state of two-dimensional electron gas well describes the experimental results.

\section{Introduction}

One of the basic problems in understanding physical properties of a two-dimensional electron gas (2DEG) is to know whether the electron density $N$ is dependent on a magnetic field or not.

Useful information about the electron density $N$ may be obtained from measurements of the thermodynamic properties of a $2 \mathrm{DEG}$, the magnetization, the specific heat, and the chemical potential. Early results for the specific heat [1], the magnetization [2, 3], and the chemical potential $[4,5]$ were interpreted in $N=$ const assumption. In order to do this, it was necessary to introduce a background density of states between the Landau levels. According to [6] an effective background density of states between the Landau levels may result from a spatial inhomogeneity of $2 \mathrm{DEG}$. Interpretation of more recent measurements of the magnetization [7] allows change of $N$ in response to the changing magnetic field.

The first measurement of the chemical potential $\mu$ performed on $\mathrm{GaAs}-\mathrm{Al}_{x} \mathrm{Ga}_{1-x}$ As heterojunction at temperature $T=4.2 \mathrm{~K}$ has shown that the experimental dependence of $\mu$ on magnetic field was greatly different from the theoretical one calculated for $N=$ const. Moreover, the Hall resistance calculated from experimental data for $\mu$ had plateaus typical for the quantum Hall effect (QHE) and was in good agreement with a direct measurement [8].

In the present work measurements of the chemical potential are made at several temperatures from 2.17 to $12.3 \mathrm{~K}$ and a thermodynamic description of the results is proposed. The experiment was performed many years ago but not published, because an explanation was absent.

\section{Experiment and Results}

The modulation-doped sample was grown by molecular beam epitaxy method on a single-crystal semi-insulating GaAs substrate in the following sequence: undoped GaAs; $0.8 \mu \mathrm{m}$; undoped GaAs- $\mathrm{Al}_{x} \mathrm{Ga}_{1-x} \mathrm{As} ; 10 \mathrm{~nm}$; GaAs- $\mathrm{Al}_{x} \mathrm{Ga}_{1-x}$ As doped with $\mathrm{Si}$ to a concentration $5 \cdot 10^{17}-10^{18} \mathrm{~cm}^{-3}$; $50 \mathrm{~nm}$; undoped GaAs; $20 \mathrm{~nm}$.

The method to measure the change of the chemical potential $\Delta \mu=\mu(H)-\mu(0)$ is based on the determination of the change in charge on the measuring capacitor consisting of the sample under investigation and reference electrode. The idea is closely related to Lord Kelvin's investigations of the contact potential difference. Let us make a capacitor from two different metals and short its plates with an electrometer (see insert in Figure 1). The measured charge will be equal to $Q=C \Delta \varphi$, where $C$ is the capacitance and $\Delta \varphi$ is the the contact potential difference between the plates (equal to the difference between their work functions). In general, the contact potential difference differs from the difference of the chemical potentials by the magnitude of the potential difference of the double charged layers present at the surface of bulk metals. If the influence of a magnetic field on the capacitance $C$ and on the work function of the reference 


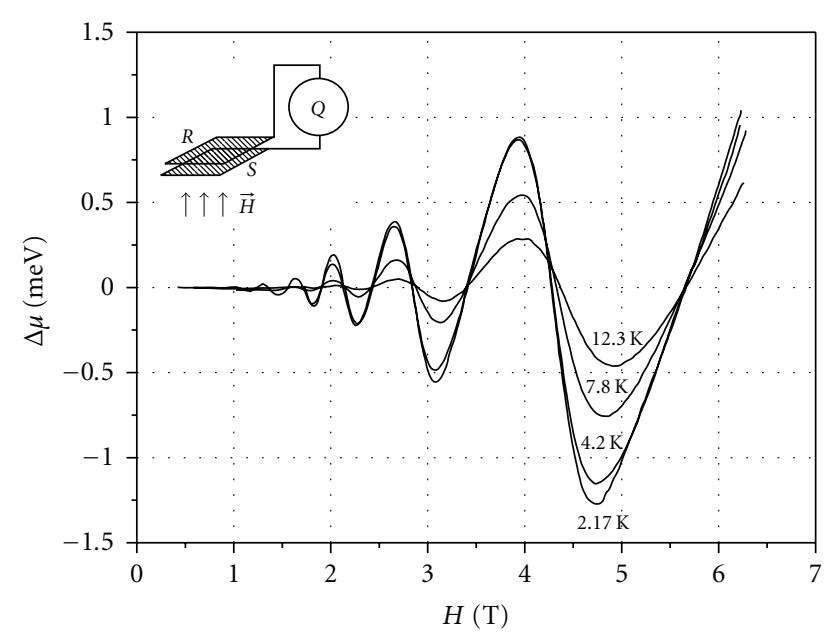

Figure 1: Change of the chemical potential measured at different temperatures. Insert: principle of the measurement ( $S$ : sample, $R$ : reference electrode, and Q: electrometer).

electrode is negligibly small, then $\Delta Q=C \Delta \mu / e$, where $e$ is the elementary electronic charge.

The reference electrode was made from bronze. The sample with area $1 \mathrm{~cm}^{2}$ was insulated from the reference electrode with thin capacitor paper; resulting capacitance was about $100 \mathrm{pF}$. The measuring capacitor was mounted at the end of the coaxial line connected to the electrometer. The coaxial line was built with sapphire insulators, and the input resistance of the electrometer was greater than $10^{16} \mathrm{Ohm}$. Calibration of the measuring capacitor was performed in a way correspondent to the classical definition of the electrostatic capacitance, namely, the charge on the capacitor was plotted as a function of slowly increasing and decreasing voltage $V(t)$ applied to the sample $(d V / d t<1 \mathrm{mV} / \mathrm{s})$. The capacitance was determined from a slope of the obtained graph. This calibration was done at different values of applied magnetic field $H$ including, of course, the quantum Hall plateaus regions. No change in the capacitance was observed within $1 \%$ accuracy.

The temperature was measured with a thermocouple $\mathrm{Cu}$ $\mathrm{Cu}(0.01 \% \mathrm{Fe})$ and stabilized with a precision of $0.05 \mathrm{~K}$.

The experimental results for $\Delta \mu$ are depicted in Figure 1. The chemical potential oscillates and amplitude of oscillations decreases with increasing temperature.

The behavior of the chemical potential calculated for $N=$ const is shown in Figure 2. These dependencies are numerical solutions of an equation for the density of $2 \mathrm{DEG}$ (spin-splitting is neglected):

$$
N=\sum_{n} \int_{0}^{\infty} D\left(\varepsilon, \varepsilon_{n}\right)\left[1+\exp \left(\frac{\varepsilon-\mu}{k_{B} T}\right)\right]^{-1} d \varepsilon
$$

with a Gaussian broadened Landau levels

$$
\begin{gathered}
D\left(\varepsilon, \varepsilon_{n}\right)=\frac{e H}{\sqrt{\pi} c h \Gamma} \exp \left[-\frac{\left(\varepsilon-\varepsilon_{n}\right)^{2}}{2 \Gamma^{2}}\right], \\
\varepsilon_{n}=\left(n+\frac{1}{2}\right) \frac{e H \hbar}{m^{*} c} .
\end{gathered}
$$

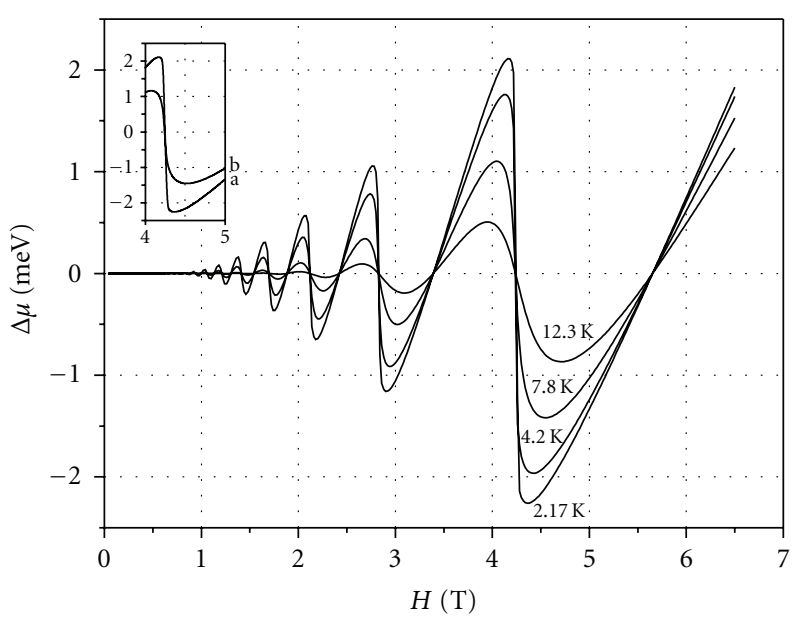

Figure 2: Change of the chemical potential calculated for $N=$ const. Insert: results for $T=2.17 \mathrm{~K}$ and $\Gamma=0.56 \mathrm{meV}$ (a), $\Gamma=$ $1.2 \mathrm{meV}$ (b).

The incoming parameters were taken as follows: the effective mass $m^{*}=0.068 \mathrm{~m}$, the electron density $N=4.1$. $10^{11} \mathrm{~cm}^{-2}$, and the broadening $\Gamma=0.56 \mathrm{meV}$. The electron density was determined from the period of oscillations in reciprocal magnetic field. The effective mass and broadening were obtained from the cyclotron resonance line position and width measured on this sample at $T=2 \mathrm{~K}$ and $\lambda=119 \mu \mathrm{m}$.

From a comparison between Figures 1 and 2 we find that the assumption $N=$ const is broken at all temperatures. By doubling the Landau level broadening it is possible to adjust the calculated amplitude of oscillations of the chemical potential to the measured one, but the shape of calculated curve remains strongly different from the experiment (see insert in Figure 2). Thus results calculated for $N=$ const differ from experiment not only quantitatively but qualitatively.

Similar disagreement between calculated for $N=$ const dependence and measured one was observed in de Haasvan Alphen effect experiments $[2,3,7]$ and in studies of the chemical potential $[5,8]$.

We also tried to approximate calculated at $N=$ const dependencies of the chemical potential on a magnetic field to those obtained experimentally by introducing an energyindependent background density of states $D=x D_{0}$ where $D_{0}=m^{*} / \pi \hbar^{2}=2.84 \cdot 10^{10} \mathrm{meV}^{-1} \mathrm{~cm}^{-2}$ is the density of states at $H=0[6]$ :

$$
N=x D_{0} \mu+(1-x) \sum_{n} \int_{0}^{\infty} D\left(\varepsilon, \varepsilon_{n}\right)\left[1+\exp \left(\frac{\varepsilon-\mu}{k_{B} T}\right)\right]^{-1} d \varepsilon .
$$

Visual agreement with the experiment was obtained for $D=0.5 D_{0} \simeq 1.4 \cdot 10^{10} \mathrm{meV}^{-1} \mathrm{~cm}^{-2}$ (Figure 3). This value of the background density of states is much larger than theoretical one $D \approx 0.3 \cdot 10^{10} \mathrm{meV}^{-1} \mathrm{~cm}^{-2}$ estimated in the $\mu$-Gaussian statistical model [6] and experimental upper limit for $D=0.036 D_{0}$ is deduced directly from the photoluminescence spectra [9]. 


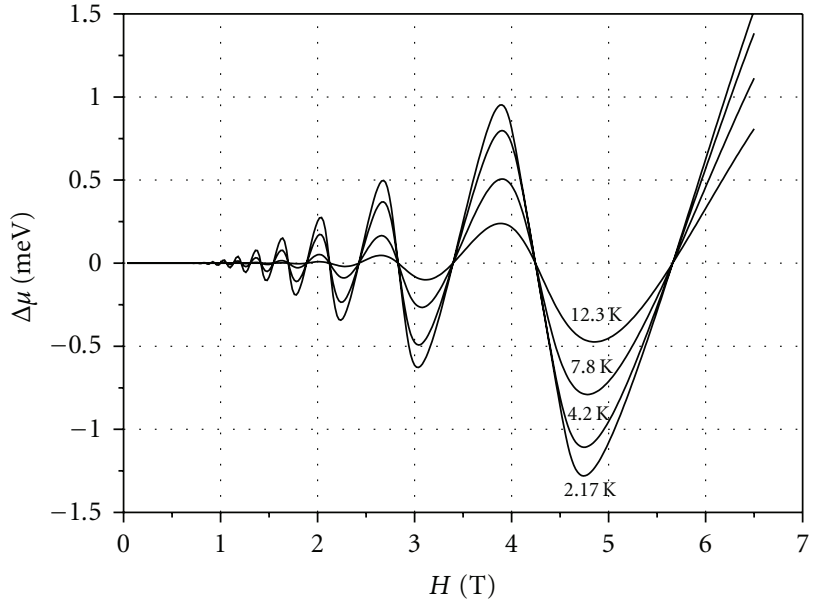

FIGURE 3: Change of the chemical potential calculated for $N=$ const with the background density of states $D=0.5 D_{0}$.

Thus assumption of $N=$ const is of little use for explanation of experimental dependencies of the chemical potential.

\section{Discussion}

Recently we have proposed a thermodynamic approach to the magnetostriction and successfully applied it for explanation of the experiments performed with nickel [10], terbium molybdate [11], and manganese fluoride [12]. These results for 3-dimensional system gave an idea to apply thermodynamics for explanation of the behavior of the field and temperature dependencies of the chemical potential $\mu$ measured on $\mathrm{GaAs}-\mathrm{Al}_{x} \mathrm{Ga}_{1-x}$ As heterojunction.

The thermodynamic description is based on the fact that magnetic field does not perform any work. The Lorentz force acting on an electric charge in magnetic field is directed perpendicular to its velocity. Free spins $\mathbf{s}$ precess around the direction of magnetic field $\mathbf{H}$ keeping an angle between $\mathbf{s}$ and $\mathbf{H}$ independent of the field strength. Net magnetic moment of the sample arises only due to the "friction" of spins with the environment (relaxation effects).

Thus for 2DEG subjected to a magnetic field the first law of thermodynamics is the same as without the field:

$$
\Delta U=T \Delta S+\alpha \Delta A,
$$

where $\Delta U=U(H)-U(0)$ is the change of the energy, $\Delta S=S(H)-S(0)$ is the change of the entropy, $\alpha$ is the surface tension, and $A$ is the area. Typical for two-dimensional systems term $\alpha \Delta A$ corresponds to $-p \Delta V$ in three dimensions [13]. Recalling definition of the Gibbs energy $G(=N \mu)=$ $U-T S-\alpha A$, we obtain, for $T=$ const and the unit area, $\Delta G=-\Delta \alpha$ or

$$
\Delta(N \mu)=-\Delta \alpha
$$

This is the thermodynamic equation of state of a 2DEG.

Appearance of the Gibbs energy is compatible with the experimental fact that both thermodynamic parametersthe chemical potential $\mu$ and the electron density $N$-are

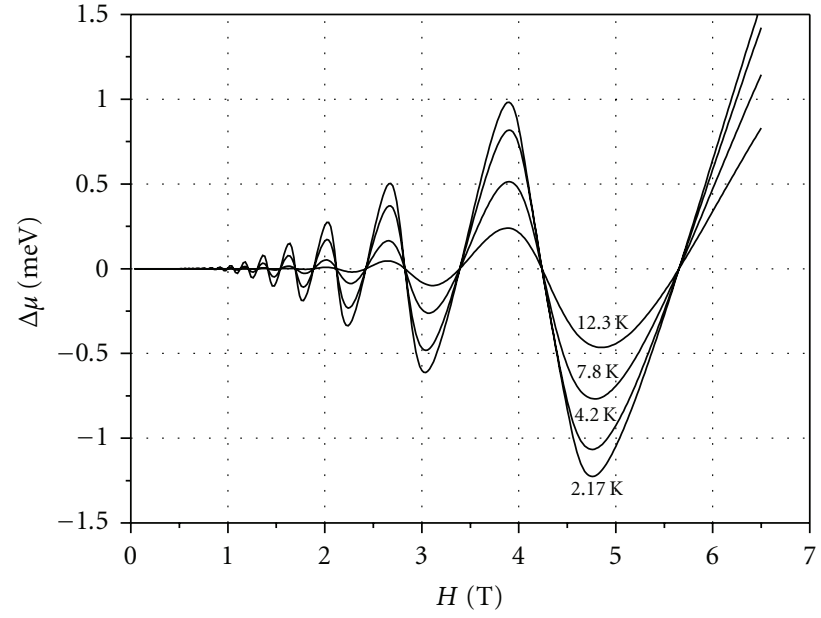

Figure 4: Change of the chemical potential calculated for $N \mu=$ const.

functions of a magnetic field. For example, the change of the free energy $\Delta F$ is equal to the work done at fixed $T, A$, and $N$ and the change of the thermodynamic potential $\Delta \Omega$ to the one done at fixed $T, A$, and $\mu$ [13]. The change of the Gibbs energy $\Delta G$ is equal to the work done at constant $T, \alpha$ and the term $\Delta \alpha$ adds the work produced by a change of the surface tension $\alpha$.

Because $U(0)=N(0) \mu(0) / 2, S(0)=\left(\pi^{2} / 3\right) N(0) T / \mu(0)$, $\alpha$ is negative, which is a natural consequence of the electronelectron repulsion. We know nothing about its dependence on a magnetic field and temperature-this problem should be elaborated by a microscopic theory. Assuming that for $i>2$ change of the surface tension $\Delta \alpha \simeq 0$ (it is difficult to imagine that the electron's repulsion is dependent on relatively low magnetic field when electron trajectories are well overlapped, because for a given $i$ the cyclotron orbit area $\left.S_{i}=i / 2 N\right)$ we calculated the dependencies $\Delta \mu(H)$ shown in Figure 4. As shown in Figure 5 the calculated Hall resistance has the plateaus that are typical of the QHE.

Figures 1 and 4 are very similar. Now we understand that experimental curves contain artifacts caused by too fast field sweep (about $0.5 \mathrm{~T} /$ minute). Due to the large time constant of the electrometer (an old model with a vibrating capacitor was used), oscillations of $\Delta \mu$ in field below $1.5 \mathrm{~T}$ were suppressed. Else, fast field sweep results in hysteresis of $\Delta \mu(H)$ dependencies in the regions of QHE plateaus, that was established 2 years after these measurements, were performed [14]. Hysteresis is aroused by a Hall effect involving weakly damped eddy currents induced in 2DEG upon a change in the magnetic field. This hysteresis is seen in Figure 1 on falling sections of $\Delta \mu$ : crossings of zero at $H \sim 4.3 \mathrm{~T}$ do not coincide. Besides, amplitude of oscillations at $T=2.17 \mathrm{~K}$ is smaller than it was expected. Having in mind these artifacts, the agreement between the experiment and theory should be considered as almost perfect.

Thus usual thermodynamics explains dependencies of the chemical potential on the magnetic field and temperature without any additional assumptions or adjustable parameters. 


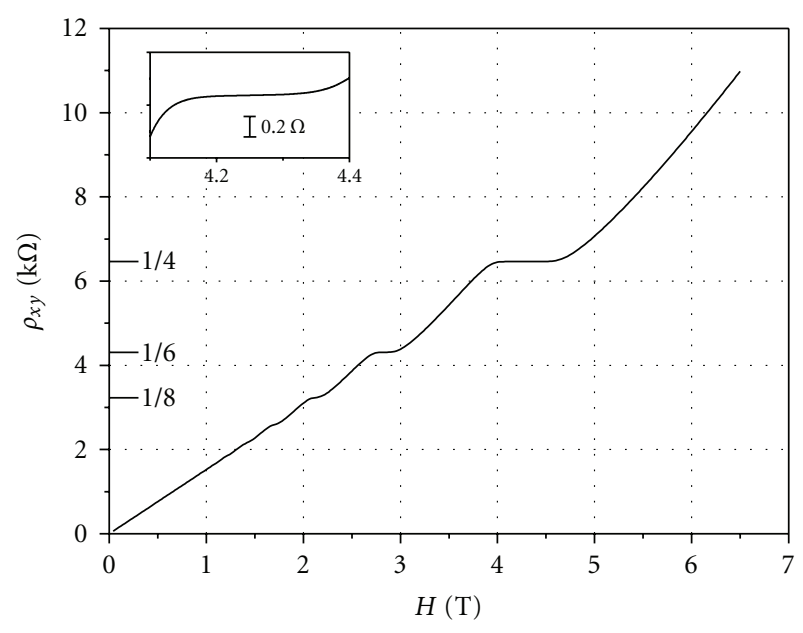

Figure 5: The Hall resistance calculated at $T=2.17 \mathrm{~K}$ for $N \mu=$ const. The horizontal strokes indicate the positions of the plateaus in units of $h / e^{2}$. Insert: $i=4$ plateau in enlarged scale.

Similarly the smoothed oscillations of the magnetization and the specific heat could be explained.

Besides the thermodynamic properties, the proposed description gives the natural explanation of the Hall plateaus observed at microwave frequencies $[15,16]$ (comparable with the cyclotron frequency $\omega_{c}=e H / m^{*} c$ ) —the plateaus result from oscillations of the total number of electrons. (Theory of the QHE for $N=$ const predicts a critical frequency that is much smaller than $\omega_{c}[17,18]$.)

Of course, the equilibrium thermodynamics could not pretend to explain all transport properties typical for the QHE.

\section{Acknowlegment}

The author is grateful to Professor E. A. Tischenko for the cyclotron resonance measurement and to Professor V. A. Berezovets for useful discussion.

\section{References}

[1] E. Gornik, R. Lassnig, G. Strasser, H. L. Störmer, A. C. Gossard, and W. Wiegmann, "Specific heat of two-dimensional electrons in GaAs-GaAlAs multilayers," Physical Review Letters, vol. 54, no. 16, pp. 1820-1823, 1985.

[2] J. P. Eisenstein, H. L. Stormer, V. Narayanamurti, A. Y. Cho, A. C. Gossard, and C. W. Tu, "Density of states and de Haas-van Alphen effect in two-dimensional electron systems," Physical Review Letters, vol. 55, no. 8, pp. 875-878, 1985.

[3] M. P. Schwarz, M. A. Wilde, S. Groth, D. Grundler, C. Heyn, and D. Heitmann, "Sawtoothlike de Haas-van Alphen oscillations of a two-dimensional electron system," Physical Review B, vol. 65, no. 24, Article ID 245315, 9 pages, 2002.

[4] V. M. Pudalov, S. G. Semenchinskii, and V. S. Edel'man, "Oscillations of the chemical potential and the energy spectrum of electrons in the inversion layer at a silicon surface in a magnetic field," Zhurnal Eksperimentalnoi i Teoreticheskoi Fiziki, vol. 89, no. 5, pp. 1870-1882, 1985, Soviet PhysicsJETP, vol. 62, no.5, pp.1079-1086, 1985.
[5] A. M. Dabiran, R. T. Zeller, F. F. Fang, S. L. Wright, and P. J. Stiles, "Electrochemical potential oscillations of the twodimensional electron gas in GaAs/AlGaAs heterostructures in high magnetic fields," Surface Science, vol. 196, no. 1-3, pp. $712-718,1988$.

[6] R. R. Gerhardts and V. Gudmundsson, "Statistical model for inhomogeneities in a two-dimensional electron gas implying a background density of states between Landau levels," Physical Review B, vol. 34, no. 4, pp. 2999-3002, 1986.

[7] A. Usher, M. Zhu, A. J. Matthews et al., "Equilibrium magnetization measurements of two-dimensional electron systems," Physica E, vol. 22, no. 1-3, pp. 741-744, 2004.

[8] V. I. Nizhankovskii, V. G. Mokerov, B. K. Medvedev, and Yu. V. Shaldin, "An investigation of the effect of a magnetic field on the chemical potential of electrons in bismuth and in a GaAs- $\mathrm{Al}_{x} \mathrm{Ga}_{1-x}$ As heterojunction," Zhurnal Eksperimentalnoi $i$ Teoreticheskoi Fiziki, vol. 90, no. 4, pp. 1326-1335, 1986, Soviet Physics-JETP, vol. 63, no. 4, pp. 776-781, 1986.

[9] M. S. Skolnick, K. J. Nash, S. J. Bass, P. E. Simmonds, and M. J. Kane, "Photoluminescence study of the density-of-states between Landau levels in the quantum hall effect system," Solid State Communications, vol. 67, no. 6, pp. 637-641, 1988.

[10] V. I. Nizhankovskii, "Classical magnetostriction of nickel in high magnetic field," European Physical Journal B, vol. 53, no. 1 , pp. 1-4, 2006.

[11] V. I. Nizhankovskii, "Magnetostriction of terbium molybdate in high magnetic field," European Physical Journal B, vol. 71, no. 1, pp. 55-57, 2009.

[12] V. I. Nizhankovskii, "Magnetostriction of $\mathrm{MnF}_{2}$ in high magnetic field," European Physical Journal B, vol. 78, no. 4, pp. 449-453, 2010.

[13] L. D. Landau and E. M. Lifshitz, Statistical Physics. I, vol. 5 of Course of Theoretical Physics, chapter 2, 15, Pergamon Press, Oxford, UK, 3rd edition, 1980.

[14] V. I. Nizhankovskii, B. K. Medvedev, and V. G. Mokerov, "Chemical potential and $g$-factor of a 2D electron gas in a strong magnetic field," Zhurnal Eksperimentalnoi i Teoreticheskoi Fiziki, Pisma, vol. 47, no. 7, pp. 343-345, 1988, Soviet Physics-JETP Letters, vol. 47, no. 7, pp. 410-413, 1988.

[15] V. A. Volkov, D. V. Galchenkov, L. A. Galchenkov et al., "Experimental observation of quantization of the Faraday rotation in a 2D electron system," Zhurnal Eksperimentalnoi $i$ Teoreticheskoi Fiziki, Pisma, vol. 43, no. 5, pp. 255-257, 1986, Soviet Physics-JETP Letters, vol. 43, no. 5, pp. 326-329, 1986.

[16] F. Kuchar, R. Meisels, G. Weimann, and W. Schlapp, "Microwave Hall conductivity of the two-dimensional electron gas in $\mathrm{GaAs}-\mathrm{Al}_{x} \mathrm{Ga}_{1-x} \mathrm{As}$," Physical Review B, vol. 33, no. 4, pp. 2965-2967, 1986.

[17] S. M. Apenko and Yu. E. Lozovik, "Quantization of the Hall conductivity of a two-dimensional electron gas in a strong magnetic field," Zhurnal Eksperimentalnoi $i$ Teoreticheskoi Fiziki, Pisma, vol. 89, no. 2, pp. 573-588, 1985, Soviet PhysicsJETP, vol. 62, no. 2, pp. 328-336, 1985.

[18] M. Pepper and J. Wakabayashi, "The frequency effect and the quantised Hall resistance," Journal of Physics C: Solid State Physics, vol. 16, no. 4, pp. L113-L117, 1983. 

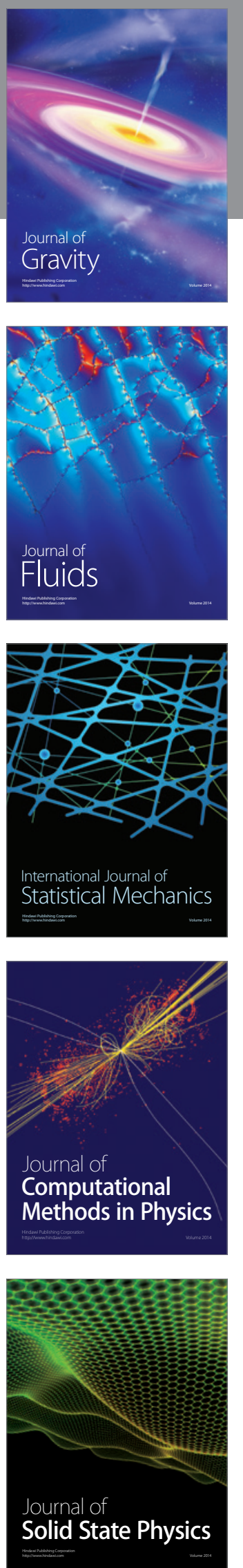

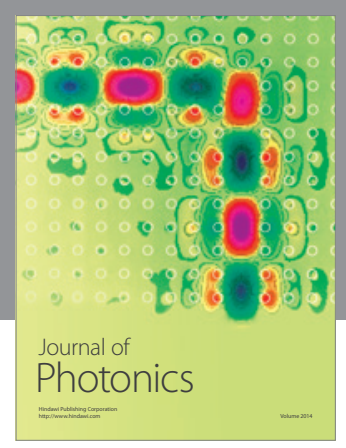

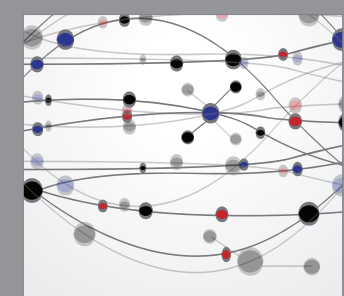

The Scientific World Journal
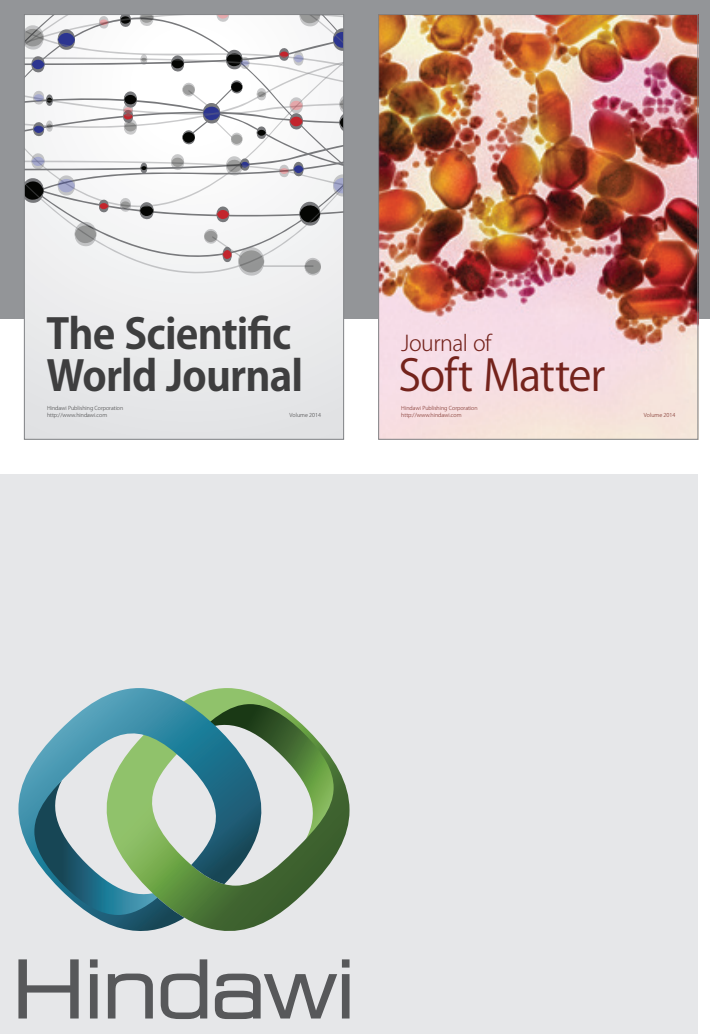

Submit your manuscripts at

http://www.hindawi.com
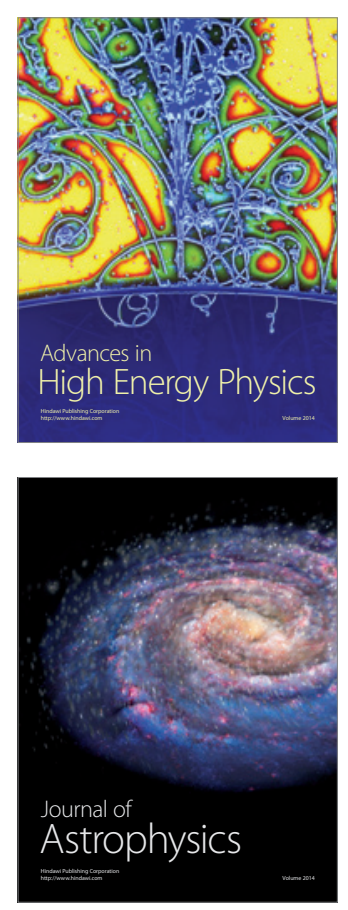
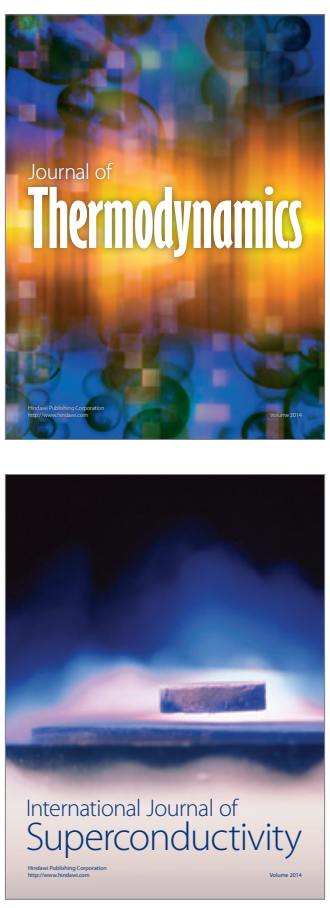
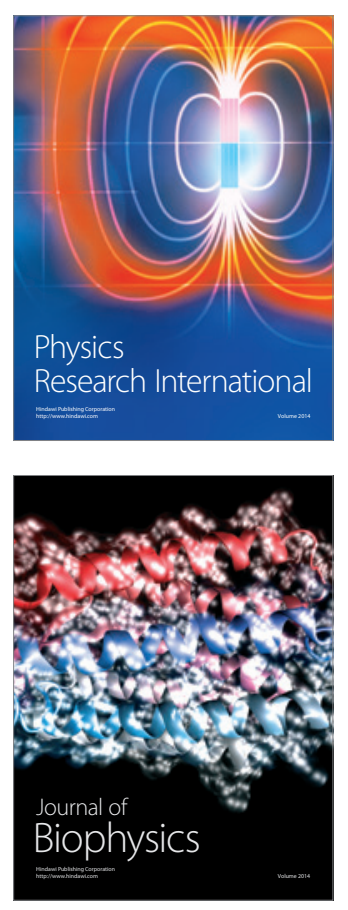
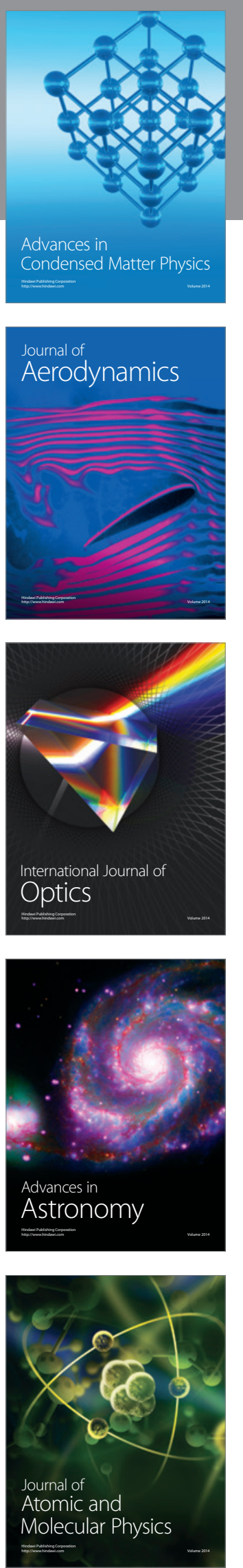